\title{
Spatial Analysis of Healthcare Facilities in the Federal Capital Territory, Abuja Nigeria
}

\author{
Mariam Sagir $^{1} \quad$ Ifeatu Enedah ${ }^{2 *} \quad$ Matthew Ono $^{3} \quad$ Johnbosco Ojiako $^{3} \quad$ Esomchukwu Igbokwe $^{3}$ \\ 1.Office of the Surveyor General of the Federation (OSGOF), Abuja, Nigeria \\ 2.Brains and Hammers Limited, Abuja, Nigeria \\ 3.Department of Surveying and Geoinformatics, Nnamdi Azikiwe University, Awka, Nigeria
}

\begin{abstract}
Over the second half of the twentieth century, the world has experienced enormous health improvements from the use of various technologies in the diagnosis, treatment, monitoring and reporting various disease. However, developing countries have benefited unequally from health gains, with many, especially in Sub-Saharan Africa (SSA), continuing to experience high mortality. This study aims to carry out a spatial analysis of healthcare facilities in the Federal Capital Territory (FCT) Abuja, Nigeria with a view of identifying the pattern of distribution of healthcare facilities. This aim was achieved by identifying and mapping the various health facilities in the FCT. The directory of Hospitals was collected from the Hospital Management Board of the FCT and the geographic location were collected using a handheld GPS device. The administrative map of Abuja and the Area Councils were digitized to obtain shapefiles for various classes of roads, railway lines, waterways and boundaries of the area councils. A geospatial database was created in the ArcMap 10.7 environment using the data collected. The locations of all healthcare facilities were plotted, and the spatial spread of healthcare facilities were analyses and queried. The healthcare facilities distribution shows a clustered pattern but underserving the study area with an average nearest neighbour ratio (NNR) for healthcare facilities in the study of $0.34(\mathrm{p}<0.001)$ and the Z-score was $-40.9(\mathrm{p}<0.001)$, the low Z-score value indicate there is a less than $1 \%$ likelihood that this clustered pattern could be the result of deliberate planning. The healthcare facilities distribution pattern suggests a disorderly and inefficient distribution of healthcare facilities. Owing to the significance of the underserving of the urban area councils by healthcare facilities, policymakers may consider placing additional healthcare facilities to reduce the stress on the available healthcare facilities and personnel.
\end{abstract}

Keywords: Spatial Analysis, GIS, Healthcare, Facilities

DOI: $10.7176 /$ JEES/11-1-06

Publication date: January $31^{\text {st }} 2021$

\section{Introduction}

Geographic Information System (GIS) plays a critical role in the decisions on where and when to intervene, improving the quality of care and accessibility of health services, finding the most cost-effective delivery modes, and protecting patient confidentiality while satisfying needs of the research community on data accessibility. GIS Applications in public health include tracking of child immunizations, conducting health policy research, and establishing health service areas and districts. GIS not only provides a way to move data from the project level to become a ubiquitous resource for an entire organization but also renders the visualization of clinical and administrative data as a spatial decision support tool. GIS serves as a common platform for the convergence of multi-disease surveillance activities (ESRI, 2011).

As towns have grown larger as a result of urbanization and industrialization, the concentration of population has, in turn, led to complicated health issues; although the importance of addressing barriers in access to health care is well documented; little has been done to improve the situation.

It is common knowledge that the general public does not know where hospitals are located, what facilities and the kinds of specialists are available. Such problems can be addressed effectively by mapping and creating relevant databases using Geographic Information Systems (GIS).

The Federal Capital Territory (FCT) Abuja, Nigeria has experienced a lot of population explosion in recent years, leading to complicated health issues, inadequate and inaccessibility of health care facilities. A proper understanding of the pattern of the spatial distribution of healthcare facilities is essential and addressing these challenges and a must for rational planning and management.

Health care Administrators in the FCT lack a functional up-to-date database of available health care facilities: the absence of comprehensive information on healthcare facilities is in FCT, Abuja is a great concern and has led to this study.

The proper location/accessibility of health care services, just like educational service, polling stations etc is an essential feature of a well-developed political region. The irregular location of health service in Nigeria has been a major obstacle for sustainable development in Nigeria.

The Nigerian health care sector has suffered tremendous neglect over the past years. Nigeria's performance is underwhelming in the health care sphere even though they hold a strategic position in Africa. Studies have 
shown that Healthcare facilities are inadequate (health centres, personnel, and medical equipment), especially in rural areas. While various reforms have been put forward by the Nigerian government to address the wide-ranging issues in the health care system, the effects of such reforms are yet to be seen or felt (Nnamuchi, 2010). According to the 2009 Communique of the Nigerian National Health Conference; health care system remains weak as evidenced by lack of coordination, fragmentation of services, the dearth of resources, including drug and supplies, inadequate and decaying infrastructure, inequity in resource distribution, and access to care and very deplorable quality of care. The conference also recognized that accurate maps were needed as a much-needed tool for assessing performance and coverage, supporting the micro-planning process and tracking areas of infectious outbreaks, especially in northern Nigeria.

In December of 2011, the National Primary Health Care Development Agency (NPHCDA) of Nigeria. (NPHCDA) and its Global Polio Eradication Initiative (GPEI) partners, with support from the Bill \& Melinda Gates Foundation, embarked on an ambitious project to address these critical needs by creating geographic information system (GIS) based maps for 8 northern states, which accounted for about $75 \%$ of communicable disease outbreak in 2010-2012 (Egwari, 2013).

GIS facilitates mapping, visualizing, monitoring, retrieving, analysing, and modelling the geo-referenced data with high accuracy (Palaniyandi, 2015). It is used for mapping and for monitoring ongoing disease control programs.

In Nigeria, the use of GIS has not been fully integrated into the public health sector. Also, inadequate data impedes researchers from fully delving into problems experienced within this sector because data is the bedrock of any analysis done with a GIS.

The most common use of a GIS is to produce data visualizations in the form of maps; the idea being that the visualization is communicating something that might not be obvious from the raw data alone.

WHO (2007) reported that access to health care services and delivery centres is concerned with the ability of a population to obtain a specified set of health care services. The major application of public health mapping is the development and making available maps to identify locations of health services; in other word health services mapping. Health services mapping includes identification of patterns of settlement, type of services concerning human settlements, and the proximity of primary health care centres, secondary and tertiary services. Factors such as distances between settlements and health services (hospital, primary health care centre, and emergency station), road infrastructure and types of transportation available greatly influence the accessibility of health services.

Musa et al. (2013) explored the use of GIS Mapping as a Public Health Tool; they noted that while traditional uses of GIS in public health are static and lacking real-time components, implementing a space-time animation in these instruments will be monumental as technology and data continue to grow.

Murad (2008) noted that GIS has several techniques and functions that can be used for health services planning. He focused on presenting a GIS application created to explore the demand and supply on health services in Jeddah city, Saudi Arabia. He created a health services geodatabase showing the location and characteristics of health supply and demand. The created application covers three major analytical issues. Murad (2008) defined and classified the location of health services based on the existing level of supply in Jeddah city, he also defined the location and characteristics of health demand in the same city. Furthermore, the researcher also defined the locations of patients and the actual catchment areas of health services. The defined catchment areas of health services will determine the growth and extent of the health catchment area in Jeddah city. The research also the identified the level of accessibility to health services using accessibility indicators scores. The results of this application were useful for evaluating the location of health services supply and demand in Jeddah city. The researcher concluded by stating that the created application is considered as a spatial decision support system for health planners in Jeddah city.

The report of the technical discussion by WHO (2007) highlighted the importance of health mapping, in association with health indicators, monitoring and evaluation. The report noted that health mapping as an e-health application is growing at a very rapid rate to support health systems development. It was noted that WHO has been using mapping techniques coupled with surveillance to monitor the global health situation and present it through user-friendly and modern tools such as geographic information systems.

Public health mapping utilizes the technology of geographic information systems to add value to information for public health planning and decision making. The role of health mapping has many aspects and influences the performance of health systems in many ways. It improves the ability of decision-makers, planners, academicians, researchers and health care professionals to organize and link thematic and spatial datasets. It provides the ability to create relations between datasets that may seem unrelated without using the geographical dimension. These links help in discovering and creating new health knowledge which can be translated into action or policies. Mapping enables professionals to understand complex spatial relationships visually, and as planning has an element of informed prediction, mapping can be a powerful tool for forecasting and trend analysis.

WHO (2007) in a technical paper in Regional Committee of Eastern Mediterranean reported that Health mapping is most valuable in the analysis of demographic data and its distribution over geography; disease 
distribution; distribution of high-risk groups; site selection and location of health services; hydrographical and water supply and sanitation systems; hydrologic modelling and water resources management and delivery; emergency preparedness and disaster management; identification of hazardous sites and disaster-prone locations (natural or man-made); health services centres and distribution and categorization of human resources.

\section{Materials and Method}

\subsection{Study Area}

The study area is Abuja, the Federal Capital Territory (FCT) of Nigeria. The FCT stretches across approximately 8,000 square kilometres with a geographic location of latitude $7^{\circ} 25^{\prime} \mathrm{N}$ and $9^{\circ} 20^{\prime} \mathrm{N}$ and longitude $5^{\circ} 45^{\prime} \mathrm{E}$ and $7^{\circ} 39^{\prime} \mathrm{E}$. It is bordered on the north by Kaduna state, on the west by Niger state, on the east by Plateau state, and on the South-west by Kogi. The geographic location of Abuja is shown in figure 1. The FCT is made up of three urban Area councils, namely Bwari, Gwagwalada, Abuja Municipal Area Council (AMAC) and three rural area councils namely: Abaji, Kwali and Kuje.

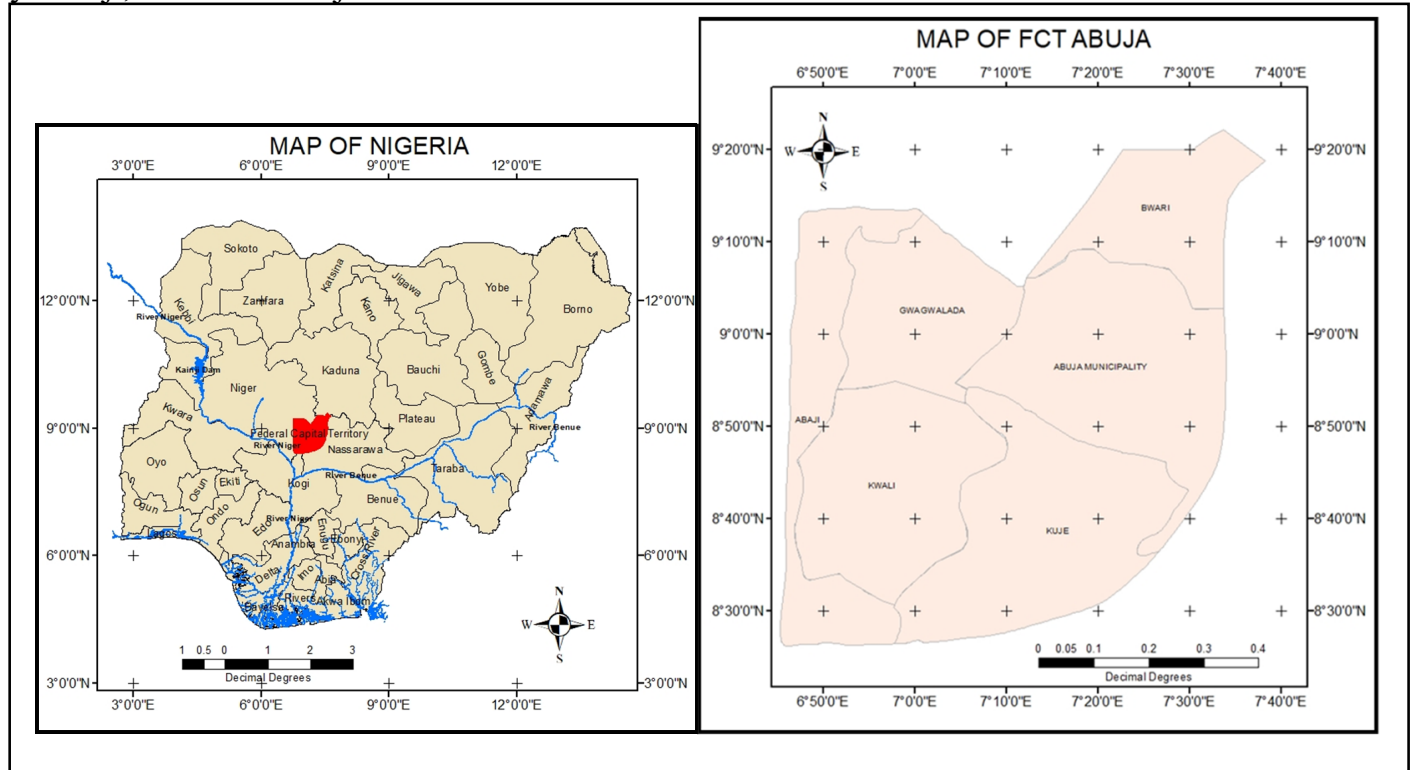

Figure 1. Map of Nigeria and Map of the study area

\subsection{Data Type and Acquisition}

i. The administrative maps of Nigeria, Abuja and the area councils were sourced from the office of Abuja Geographic Information System (AGIS).

ii. The geographic locations of the public and private healthcare facilities in Abuja were acquired by collecting their coordinates using handheld GPS equipment with support from the local communities. These health care facilities of interest include:

a. Traditional/spiritual healing centres and Traditional birth attendants

b. Patent medicine store and Pharmacy

c. Tertiary hospitals

d. Secondary hospitals

e. Primary hospitals and Primary health centres

iii. Attribute data: This includes non-spatial descriptive information of the health care facilities which includes area council, ward, name and type of facility were sourced from the field as well as from statutory organisations.

iv. Directory of Hospital/health facilities and bed spaces in Abuja were sourced from the Health Department of the Federal Capital Development Administration (FCDA).

v. The total number of Medical personnel in Abuja were sourced from Health Department of FCDA, the Nigerian Medical Association and Hospital management board of the FCT.

vi. Demographic data of the study area was sourced from the website of the National Population Commission.

2.2.1Hardware Requirements

The following hardware was used to fulfil the aim and objectives of this study:

a. A handheld GPS receiver (Garmin eTrex 64); this was used for capturing the location (x, y and z) of hospitals and health facilities.

b. A Computer (Laptop) system with the following configuration - Hewlett Packard Pavilion HDX, 
Processor - Intel ${ }^{\circledR}$ core $^{\mathrm{TM}} 2$ Duo CPU T9300@2.50Ghz 15.20GHz, Memory (Ram) - 4.00G, Memory (HDD) - 500GB

c. HP Deskjet 1515 printer for printing of reports and coloured images and maps in A4 size)

\subsubsection{Software Requirements}

The following software was used for achieving the goal of this project:

i. ArcGIS 10.7 handles multiple tables and relates them to each other with ease and allows manipulation and query using appropriate commands. This was used for geospatial database creation, thematic mapping and visualisation of both communicable diseases and healthcare facilities.

ii. Microsoft Offices; Microsoft word was used for typesetting of the reports, Microsoft Excel was used for the creation of tables, and Microsoft Visio was used for creating charts.

\subsection{Methodology}

To achieve the aim and objective of this study, the administrative map of Abuja and the Area Councils were digitized to obtain shapefiles for various classes of roads, railway lines, waterways and boundaries of the area councils. The geographic locations of healthcare facilities collected were formatted and arranged in an Excel file (table) and saved in the 97-2003 workbook (*.xls) file format (format accepted by ARCGIS); for easy manipulation. The table contains information on the $\mathrm{x}$, $\mathrm{y}$ coordinates (eastings, northings or Latitude, longitude) as well as attribute information on the facilities. ArcMap allows the addition of such tabular data that contains geographic locations in the form of $\mathrm{x}, \mathrm{y}$ coordinates to map. The "Add X, Y data" in 'Add data tool' was used to plot the locations of the healthcare facilities. A geospatial database was created using all the data collected.

\section{Results and Discussion}

The geospatial database created was used in achieving this objective of this paper. Various classes of Healthcare facilities were identified in the study area and mapped accordingly. The maps created can serve as decision support system (DSS) for healthcare facilities in the FCT.

\subsection{Spatial distribution of healthcare facilities in FCT, Abuja}

Healthcare facilities in the FCT, Abuja are grouped based on the type of service rendered and the professional rendering the service. The various categories of Healthcare facilities that dot the study area mapped accordingly; the categories include:

i. $\quad$ Primary healthcare facilities

ii. $\quad$ Secondary healthcare facilities

iii. Tertiary healthcare facilities

iv. Traditional healthcare facilities

v. Patent medicine store and Pharmacy 
Figure $3.1 \mathrm{a}, \mathrm{b}, \mathrm{c}$, and d show the various classes of health care facilities in the study area.
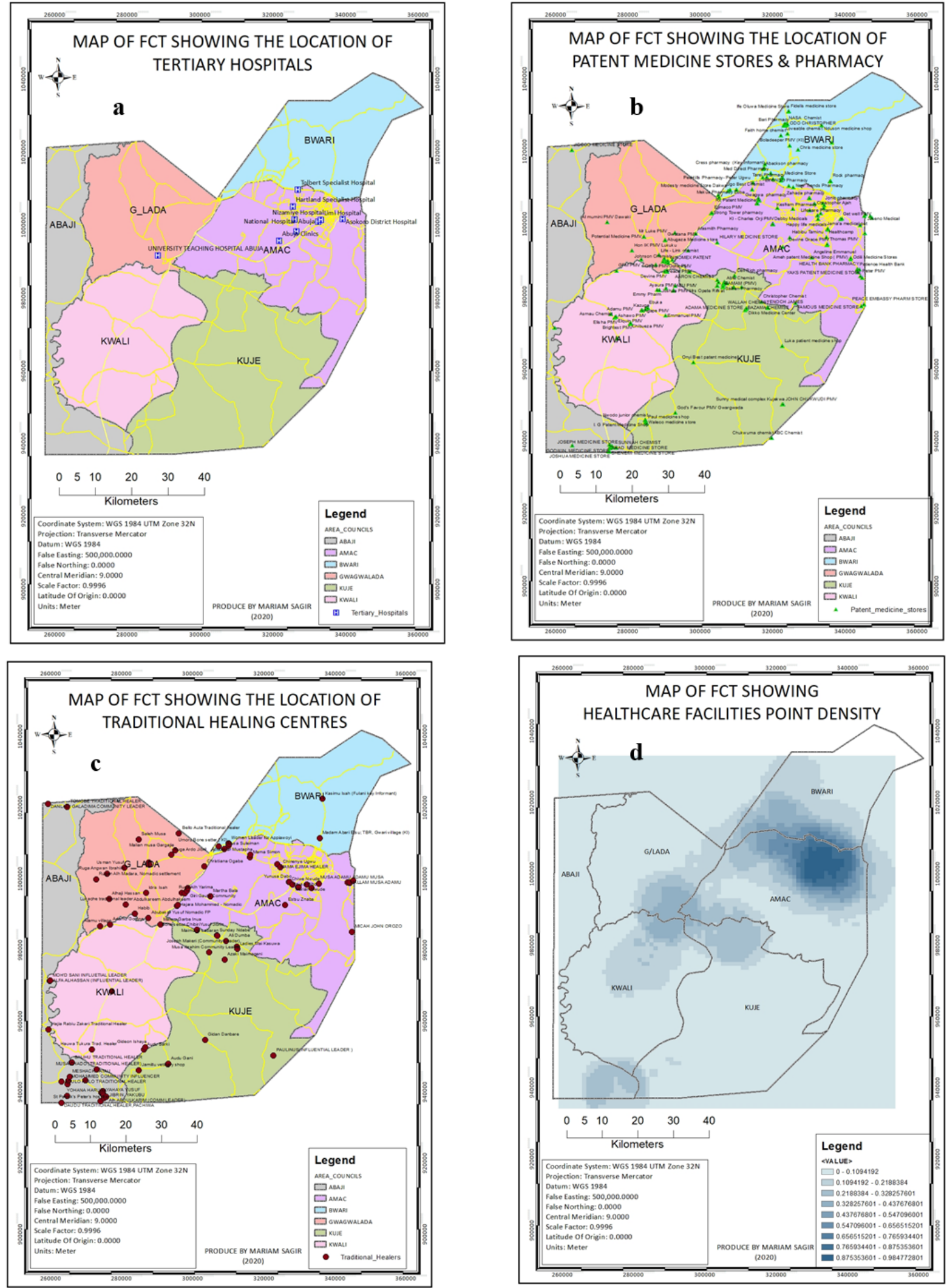

Fig 3.1 (a) Tertiary healthcare facilities, (b) Patent medicine store and Pharmacy, (c)Traditional healing centre, (d) Spatial distribution of health care facilities.

Available data indicate that there are more healthcare facilities in the area councils classified as urban area councils which include AMAC, Bwari and Gwagwalada than these classified as rural area councils which are Kuje, Abaji, and Kwali. 
To assess the pattern of the spatial distribution of Healthcare facilities in the FCT, the Average Nearest Neighbour tool used in the ArcGIS 10.7 environment. The findings indicate that the healthcare facilities are spatially clustered across the FCT (see Figure 4.150). The average nearest neighbour ratio (NNR) was 0.34 ( $\mathrm{p}<$ $0.001)$ and the $\mathrm{Z}$-score was $-40.9(\mathrm{p}<0.001)$. Due to the low Z-score value, there was less than $1 \%$ likelihood that this clustered pattern could be the result of deliberate planning.

Generally, the concentrated spatial pattern of the distribution of healthcare facilities tends to be in the area councils classified as urban area council; these include AMAC, Bwari and Gwagwalada. The spatial pattern further suggests a disorderly and inefficient distribution of healthcare facilities. For example, eight out of the nine Tertiary Hospitals in the FCT are in the Abuja Municipal area council (AMAC) and the last one located in the Gwagwalada area council. The other four area councils are without any tertiary hospital. In the same manner, a fewer number of healthcare facilities are in rural area councils which includes Abaji, Kuje and Kwali although these area councils by far have the lowest population.

\subsection{Level of Healthcare Facilities coverage}

In mapping healthcare facilities, an area can be either over or under-covered. Comparatively, the under-coverage area is defined as areas with public health density within the lowest quantile of all health facility densities (public and private) across the area councils. In the same vein, the over-coverage area is defined as area councils with a public health density within the highest quantile of all health facilities densities across all districts. Worthy of note is that the under- and over-coverage analysis is mostly a comparative analysis and should not be regarded as resource availability analysis (Schwanke Khilji et al. 2013).

Spatial distribution of Healthcare facilities was analysed using queries and point density at area council level to identify the area councils of relatively under and over coverage. The output of this query (see Figure 2 and 3 ) revealed that AMAC has the highest number of Healthcare facilities (about 404 facilities) while Abaji has the lowest number of facilities (about 89 facilities)

The healthcare facilities point density as shown in Figure 7 displayed the heaviest clustering around AMAC (Federal Capital City centre) and Bwari area council; another clustering around the boundaries of Gwagwalada, Kuje, AMAC and Kwali.

\subsection{Hospital to Population Ratios}

Providing an adequate number of healthcare facilities at each administrative unit (e.g. area council or local government) is undoubtedly the core concern of most healthcare planners. Analysing healthcare facility to population ratio is very important to enable policymakers to determine how best to meet the basic healthcare needs of any population. Healthcare facility to population ratios can differ significantly between rural and urban areas as well as across urban areas and rural areas.

Healthcare facilities must be geographically adeptly spread according to population concentration and density. Health facility distribution per 10,000 population is a useful standardized indicator in measuring levels of health services accessibility by the population living within a geographic administrative unit. This method allows for a comparison between spatial units and identification of gaps in health service coverage across the study area (WHO 2010). The indicator of the distribution of healthcare facilities per 10,000 population was calculated simply by $10,000 \times($ Number of public health facilities/Total population in each area council). The output of this analysis is presented in Table 3.1

Table 3.1 Hospitals to Population ratio

\begin{tabular}{|l|l|l|l|l|l|}
\hline S/N & Area Council & $\begin{array}{l}2018 \\
\text { Estimated } \\
\text { Population }\end{array}$ & $\begin{array}{l}\text { Total no of Healthcare } \\
\text { facilities (Hospitals only) }\end{array}$ & $\begin{array}{l}\text { Percentage } \\
\text { of total } \\
\text { hospitals }\end{array}$ & $\begin{array}{l}\text { Hospitals to } \\
\text { population ratio }\end{array}$ \\
\hline 1 & Abaji & 151,055 & 89 & 9 & 5.8919 \\
\hline 2 & AMAC & $1,999,657$ & 412 & 40 & 2.0604 \\
\hline 3 & Bwari & 590,584 & 173 & 16 & 2.9293 \\
\hline 4 & Gwagwalada & 408,583 & 127 & 12 & 3.1083 \\
\hline 5 & Kuje & 250,461 & 138 & 13 & 5.5098 \\
\hline 6 & Kwali & 221,975 & 103 & 10 & 4.6402 \\
\hline
\end{tabular}

The rural area councils have higher Hospital to population ratios because of their low population figures compared to the urban area councils. AMAC, where the Federal Capital City (FCC) is located, has the lowest hospital to population ratio, this because despite having almost $40 \%$ of the total number of hospitals in the area council, its population is equally very high compared to other area councils

\section{Conclusion and Recommendation}

This research has made several contributions to the existing body of knowledge in the area of mapping of healthcare facilities in Abuja, Nigeria. The research contributes to knowledge includes: 
The comprehensive geospatial database of healthcare facilities can help the relevant stakeholders in monitoring and regulating the operations and certification of the so-called healthcare facilities. The comprehensive geospatial database of healthcare facilities can also be of immense benefit to the collection of accurate data on health statistics such birth rate, death rate and diseases occurrences as the geolocation of the facilities where the statistics are generated is already known.

The provision of healthcare facilities plays a vital role in ensuring healthcare delivery and the well-being of any community. Therefore, the provision of healthcare facilities and services based on population location is important and should be a matter of public policy for the government.

The findings of this paper has undoubtedly exposed that there is a cluster pattern of healthcare facility distribution in the urban area councils, and at the same, underserved by healthcare facilities. Ironically, the rural area councils which have fewer healthcare facilities and expectedly lower population, have higher healthcare facility to population ratio. Thus, medical personnel working in the urban area council will be overworked.

Owing to the significance of the underserving of the urban area councils by healthcare facilities, policymakers may consider placing additional healthcare facilities to reduce the stress on the available healthcare facilities and personnel. The policy-makers should consider increasing the number of healthcare facilities in the urban area councils, the new healthcare facilities should be required to be located and distributed according to population.

The Hospital Management Board should ensure the collection of data on private healthcare facilities as there is no such data currently. They should also develop and ensure an up-to-date database of all healthcare facilities in the FCT; not only the orthodox facilities but also unorthodox facilities. Finally, policymakers can adopt the geospatial database created in this study for monitoring and regulating healthcare facilities in the study area.

\section{References}

Egwari, L. O., (2013). Health Education in the Control and Prevention of Communicable and Non-Communicable Diseases in Nigeria: Article from the Covenant University - Igbinedion. $1-31$.

Environmental Systems Research Institute, (Esri), (2011). Geographic Information Systems and Environmental Health - Incorporating Esri Technology and Services. White paper, USA.

Murad, A. A. (2008). "Defining Health Catchment Areas in Jeddah City, Saudi Arabia: An Example Demonstrating the Utility of Geographical Information Systems." Geospatial Health 2 (2): 151-160. doi:10.4081/gh.2008.239.

Musa, J. G., Chang, P., Sylk, T., Bavley, R. and Hoven, C. (2013). Use Of GIS Mapping As A Public Health Tool-From Cholera To Cancer In Health Services. Insight 6, (111 - 116) doi: 10.4137/HSI.S10471

Nnamuchi O. (2010). The right to health in Nigeria. 'Right to health in the Middle East' project, Law School, University of Aberdeen. Available from: http://www.abdn.ac.uk/law/hhr.shtml. (May 2018)

Palaniyandi, M. (2015). GIS for rapid epidemiological mapping and healthcare management with special reference to filariasis in India. International Journal of Medical Science and Public Health 4:8

World Health Organization. Regional Office for the Eastern Mediterranean. (2007). Use and potential of geographic information systems for health mapping in the Eastern Mediterranean Region. https://apps.who.int/iris/handle/10665/122558 (May 2018) 